\title{
A Type-2 Fuzzy Approach to Driver-Automation Shared Driving Lane Keeping Control of Semi- autonomous Vehicles under Imprecise Premise Variable
}

\author{
Yue Liu \\ Beihang University \\ Qing Xu \\ Tsinghua University \\ Hongyan Guo \\ Jilin University
}

Hui ZHANG ( $\sim$ huizhang285@gmail.com )

Beihang University https://orcid.org/0000-0002-2501-712X

\section{Original Article}

Keywords: Fuzzy control, interval type-2 fuzzy systems, Dstability , driver-automation shared control, autonomous vehicles

Posted Date: August 24th, 2021

DOI: https://doi.org/10.21203/rs.3.rs-814651/v1

License: (c) (1) This work is licensed under a Creative Commons Attribution 4.0 International License. Read Full License 


\title{
A Type-2 Fuzzy Approach to Driver-Automation Shared Driving Lane Keeping Control of Semi-autonomous Vehicles under Imprecise Premise Variable
}

\author{
Yue Liu, Qing Xu, Hong Yan Guo, and Hui Zhang*
}

\begin{abstract}
The driver-automation shared driving is a transition to fully-autonomous driving, in which human driver and vehicular controller cooperatively share the control authority. This paper investigates the shared steering control of semiautonomous vehicles with uncertainty from imprecise parameter. By considering driver's lane-keeping behaviour on the vehicle system, a driver-automation shared driving model is introduced for control purpose. Based on the interval type-2 (IT2) fuzzy theory, moreover, the driver-automation shared driving model with uncertainty from imprecise parameter is described using an IT2 fuzzy model. After that, the corresponding IT2 fuzzy controller is designed and a direct Lyapunov method is applied to analyze the system stability. In this work, sufficient design conditions in terms of linear matrix inequalities are derived, to guarantee the closed-loop stability of the driver-automation shared control system. Meanwhile, an $\mathcal{H}_{\infty}$ performance is studied to ensure the robustness of the control system. Finally, simulationbased results are provided to demonstrate the performance of the proposed control method. Furthermore, an existing type-1 fuzzy controller is introduced as comparison, to verify the superiority of the proposed IT2 fuzzy controller.
\end{abstract}

Index Terms-Fuzzy control, interval type-2 fuzzy systems, $\mathcal{D}$ stability, driver-automation shared control, autonomous vehicles.

\section{NOMENClATURE}

$C_{r} \quad$ Rear concerning stiffness

$C_{f} \quad$ Front concerning stiffness

$l_{f} \quad$ Distance from center of gravity to front axle

$l_{r} \quad$ Distance from center of gravity to rear axle

$l_{w} \quad$ Distance from center of gravity to wind impact center

$l_{s} \quad$ Preview distance

$n_{R} \quad$ Tire pneumatic trail

$I_{z} \quad$ Vehicle yaw inertia moment

$I_{s} \quad$ Inertia moment of steering system

$R_{s} \quad$ Steering gear ratio

$B_{s} \quad$ Steering system damping coefficient

$m \quad$ Vehicle mass

Hui Zhang is the corresponding author with the Email: huizhang285@gmail.com

Yue Liu, and Hui Zhang are with the School of Transportation Science and Engineering, Beihang University, Beijing 100091, China. e-mail: (sy1613207@buaa.edu.cn; Huizhang285@gmail.com)

Qing Xu is with Department of automotive engineering, Tsinghua University, Beijing, China

Hong yan Guo is with Colleague of automotive engineering, Jilin University, Jilin, China

\author{
$K_{d 1}$ \\ $K_{d 2}$ \\ $t_{p}$ \\ $\tau_{a}$

\begin{abstract}
Driver's proportional action about near angle
Driver's proportional action about far angle

Preview time

Driver's anticipatory time
\end{abstract}

\section{INTRODUCTION}

$\mathbf{V}$ EHICLE automation has attracted extensive attention from academia, industry and government over the past decades. It can reduce driver workload and road accidents resulted from human factors such as distraction and drowsiness, etc. However, there are still some barriers in the way to fully driving automation, such as the limitation of contemporary technology and anthropologic issue. Furthermore, some severe autonomous-driving accidents also indicate the human-off-the-loop driving is dangerous [1], [2]. It means that: although the fully driving automation is an ultimate appeal for autonomous vehicle, the human driver still plays an important role in the current stage. However, inattention of human driver does threaten the vehicle safety, and consider human driver's physical and psychological influence on the strategic, tactical and control behavior, he/she may do misoperation in complex environment. Consequently, there are many advanced vehicle assistance systems, like adaptive cruise control system (ACC), lane departure warning system (LDWS) and lane keeping assistance system (LKAS) [3], [4], that are developed to alleviate driver's burden and improve the vehicle safety. In general, these vehicle-borne auxiliary systems could undertake driving tasks independently. For example, in a circumstance where the road is longer and straighter and the destination is farther apart, ACC could follow the preceding vehicle automatically. However, these assistance systems have their dynamic and situated limitations, for instance, the bad weather condition could degrade the lead vehicle following performance of the ACC system [5]. Similarly, other advanced assistance systems may also outside their control bandwidth in the case where the external environment changes rapidly [5]. As a result, it is necessary that human driver and vehicular assistance system control the vehicle cooperatively. Therefore, there is an inevitable interaction between the human driver and the assistance system.

Taking the electric power assist system(EPAS) for instance, when human driver implements his/her control behavior on the steering wheel, EPAS could assist the driver to adjust the 
steering through an electric motor. With hands on the steering wheel, the driver could perceive that the vehicle has its own intention. It is worthy mentioning that there may be unexpected assistance in regular driving, which can disturb the driver and even be harmful to vehicle safety. Therefore, conflict between human driver and automation system is a challenge needed to be settled. Fortunately, shared control is a valid approach to weaken conflict between them [6], [7], in which human driver and automation system share the control authority of vehicle collaboratively. In fact, there is no consentaneous definition for shared control in academia, Abbink et al. proposed: in shared control, human and 'robot' are interacting congruently in a perception-action cycle to perform a dynamic task that either human or the robot could execute individually under ideal circumstances [5]. Here, the 'robot' means a designed system with a degree of 'intelligence' and 'autonomy', such as the vehicle equipped with advanced assistance systems, which can complete particular driving task independently under certain circumstances. There are some other researchers that emphasize the essence of shared control lies in the execution of actions, although 'monitor' and 'decision' can also be shared [8]. This emphasis on "low-level" execution is also central to the proposed definition of shared control for automotive domain [5]. There are two different types of shared control: directly shared control and indirectly shared control [9]. The former means the 'robot' will interpose the driver directly while the latter emphasizes that the 'robot' will compensate the driver' activity.

Shared control has been extensively studied in the last two decades. Saleh et al. [10] designed a shared control method,which was based on a closed-loop driver-vehicle-road model and the shared control law was realized through the $\mathcal{H}_{2}$-preview control method. Huang et al. [11] presented a shared framework of the driver and the semi-autonomous vehicle, in which a data-driven shared control law using robust adaptive dynamics programming was studied. Li et al. [12] proposed a driver-automation shared control strategy for obstacle avoidance, where a two-layer fuzzy strategy was introduced to address the control authority allocation issues. Erlien et al. [13] proposed a shared control framework for obstacle avoidance using two safe driving envelopes, where a model predictive control (MPC) scheme was developed to determine if the driver can ensure a safe vehicle trajectory within proposed two envelopes and when to intervene the driver. Ji et al. [14] proposed a novel stochastic game-based shared control framework to model the steering torque interaction between human driver and the intelligent electric power steering system. Li et al. [15] presented an 'indirect shared control' framework for steer-by-wire vehicles, in which a 'best-response' driver steering model based on MPC was proposed. Lu et al. [16] studied the driving performance of brain-controller vehicles which are controlled by human 'mind' through the use of a brain-computer interface. In this work, they proposed a shared control method based on the MPC strategy, to ensure the brain-control drivers have the maximum control authority. Nguyen et al. [17] studied the shared control between human driver and LKAS for lane keeping and obstacle avoidance, in which a fictive nonlinear function was proposed to describe the driver activity. They proposed a type-1 Takagi-Sugeno fuzzy method to deal with the time-varying vehicle speed. However, although the type-1 fuzzy technology is useful in describing nonlinear and/or linear parameter-varying systems [18], it cannot describe uncertainty in the membership function. Generally, the vehicle longitudinal speed in system model can be determined through the wheelspeed sensor. However, the precision of measurement is limited due to sensor performance. Usually, high precision comes with high cost, while low-cost sensor leads to inevitable measurement error. This inevitable measurement error will cause the imprecise longitudinal speed. Moreover, when the wheel is not free-rolling, the longitudinal speed obtained based on the wheel-speed sensor will also be imprecise. The imprecise longitudinal speed can cause uncertain membership function. If the uncertainty is neglected when designing controller, the performance of designed controller will be greatly affected. Fortunately, type-2 fuzzy technology is a valid method to further address uncertainty in the membership function.

The conception of type- 2 fuzzy system was proposed by Zadeh in [19]. It can be regarded as a collection of infinite number of type-1 fuzzy systems [20], [21]. Different from type-1 T-S fuzzy system, the type- 2 fuzzy system has a better property in describing uncertainties which cause difficulty to determine exact membership function [22], [23]. There are various sources of uncertainties in type-1 fuzzy system: (1) Measured signals activating type-1 fuzzy system may be contaminated by noise or other factors and therefore be uncertain; (2) Data applied to type-1 fuzzy system to modify parameters may be incomplete or fragmentary [24], [25]. All of these can generate uncertainty in the membership function. Fortunately, it can be removed by the type- 2 fuzzy technology, which has been researched and demonstrated in many literatures. Hani et al. [26] studied the autonomous mobile robot navigating in changing environment, in which a type-2 fuzzy logic technology was developed to handle uncertainties from the changing and unstructured environments. Zhang et al. [27] addressed the fault detection problem for continuous-time fuzzy semi-Markov jump systems, in which an interval type-2 fuzzy approach was utilized to solve the parameter uncertainty of system. Kumbasar [28] proposed a self-tuning zSlices-based general type-2 fuzzy PI controller to handle high levels of uncertainties and ensure the robustness of system to disturbances noise and uncertainties, where the secondary membership functions were adjusted in an online manner. Muhuri et al. [29] addressed the multi-objective reliability-redundancy allocation problem to ensure high system reliability in the presence of optimally redundant components with uncertain parameters, in which an interval type-2 fuzzy technology was utilized as type-1 fuzzy technology has limitations in representing high order uncertainties.

This paper studies the driver-automation shared steering control problem for lane keeping performance. In order to attenuate the conflict between human driver and vehicular automation system, interaction between them is necessary to be studied. As mentioned before, the driver could perceive the vehicle having its own intention with hands on the steering wheel. Apart from this, the vehicular automation also 
needs to know the driver's behavior and provide appropriate assist torque based on it. Consequently, a time-varying weighting function which is related to the driver activity is introduced here to adjust the assist torque, so that an appropriate assistance level can be ensured. Considering the uncertain driver behavior, furthermore, a two-point preview driver model is used to describe the driver's behavior. Then, a driver-automation shared driving model is obtained. As the vehicle longitudinal speed is time-varying in the driving process, the obtained model will be a linear parameter-varying model. Although type-1 T-S fuzzy theory is a valid method to address such model, it cannot remove the uncertainty in the membership function resulted from inevitable measurement error. Moreover, the introduced time-varying weighting function also contains time-variant variable, which can also cause uncertain membership function. Therefore, a type-2 fuzzy technology is studied in this work, to address uncertainty in the membership function and the controller design issue. Differing from the previous work in [30], an $\mathcal{H}_{\infty}$ performance index is introduced here, to weaken the influence of external disturbance on the control system. It is because both the lane keeping performance and the system robustness should be guaranteed. Besides, data from urban driving condition is also applied to verify effectiveness of the proposed method. The main contributions of this paper are summarized as follows:

1) A new type-2 fuzzy technology is applied to remove uncertainty in the membership function resulted from measurement error and the driver's state parameter;

2) A different control output is introduced, which considers information of both the lane keeping performance and conflict between human driver and vehicular automation system;

3) A $\mathcal{D}$-stability method is introduced to realize the pole placement and then improve transient performance of the control system.

\section{Problem Statement}

This section formulates the driver-automation shared control problem. As mentioned before, if the interaction between human driver and vehicle assistant system is ignored, it may lead discomfort to the driver and even conflict between them. To avoid conflict issues, the driver-automation shared control law for a lane keeping purpose is studied. Considering uncertain driving behavior of the driver, a two-point previewed driver model is applied to the road-vehicle model and then the driverautomation shared driving model can be obtained. Moreover, a type-2 fuzzy technology is introduced to address time-varying parameters and uncertainty in the system.

\section{A. Driver-Automation Cooperative Driving Model}

In this work, a shared steering control strategy for lane keeping performance is studied. Here, It is assumed that there are no vehicles moving into the lane on both sides of the target vehicle. Then, a lateral vehicle dynamics as shown in Fig. 1 is studied. In addition, in order to analyze the robustness of the control system, effects of external disturbances like the lateral

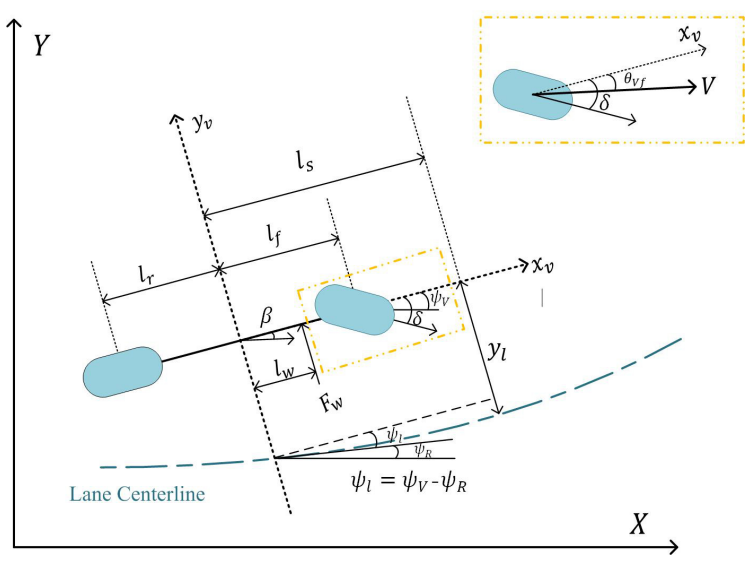

Fig. 1. Schematic diagram of vehicle-road model.

wind is considered. Therefore, the lateral translational motion and the yaw dynamics of vehicle is described as follows: [31]:

$$
\begin{aligned}
m \dot{v}_{y} & =-\frac{2\left(C_{f}+C_{r}\right)}{v_{x}} v_{y}+\frac{2\left(C_{r} l_{r}-C_{f} l_{f}\right)}{v_{x}} r-m v_{x} r \\
& +2 C_{f} \delta+F_{l}, \\
I_{z} \dot{r} & =\frac{2\left(C_{r} l_{r}-C_{f} l_{f}\right)}{v_{x}} v_{y}-\frac{2\left(C_{f} l_{f}^{2}+C_{r} l_{r}^{2}\right)}{v_{x}} r \\
& +2 C_{f} l_{f} \delta+l_{w} F_{l} .
\end{aligned}
$$

where $v_{y}$ is the lateral velocity and $v_{x}$ is the longitudinal velocity. $r$ represents the yaw rate. $m$ is the total vehicle mass. $I_{z}$ is the vehicle yaw inertia moment. $l_{f}$ shows the distance from center of gravity to front axle and $l_{r}$ is the distance from center of gravity to rear axle. $l_{w}$ indicates the distance from center of gravity to the lateral wind impact center. $C_{r}$ is the concerning stiffness of rear tires and $C_{f}$ is the concerning stiffness of front tires. $\delta$ represents the front wheel steering angle.

In this work, in order to pursue better lane keeping performance, a model needs to be defined to describe the vehicle lateral offset. As shown in Fig.1, considering the look-ahead distance, the lateral offset consists of two parts: (1) the lateral displacement resulted from vehicle motion; (2) the component originating from the look-ahead distance. As a result, the model of vehicle lateral offset is defined as follows [32]:

$$
\dot{y}_{l}=v_{y}+l_{s} r+v_{x} \psi_{l},
$$

where $y_{l}$ shows the lateral offset, $l_{s}$ is the look-ahead distance and $\psi_{l}$ is the heading error. It should be noted that the heading error is approximatively assumed to be the yaw angle. The assumption is valid in circumstance where the road curvature is too small to be neglected, which is realistic for the highway driving environment. Then, the following equation could be obtained:

$$
\dot{\psi}_{l}=r
$$

In the framework of the driver-automation shared driving, the human driver implement a torque on the steering wheel while the vehicular automation controller also provide an assistant torque to control the vehicle. Then, a desired frontwheel steering angle is obtained through the steering system. 
Considering the damping characteristic of steering system and the aligning torque resulted from front-tire lateral force, the equation of front-wheel steering angle is shown as follows:

$$
\begin{aligned}
I_{s} \ddot{\delta} & =\frac{T_{a}+T_{d}}{R_{s}}-\frac{F_{y f} n_{R}}{R_{s}^{2}}-B_{s} \dot{\delta} \\
& =\frac{2 C_{f} n_{R}}{R_{s}^{2} v_{x}} v_{y}+\frac{2 C_{f} l_{f} n_{R}}{R_{s}^{2} v_{x}} r-\frac{2 C_{f} n_{R}}{R_{s}^{2}} \delta-B_{s} \dot{\delta}+\frac{T_{a}+T_{a}}{R_{s}},
\end{aligned}
$$

where $I_{s}$ is the moment of inertia and $R_{s}$ is the gear ratio of steering system. $B_{s}$ is the damping coefficient of steering system. $n_{R}$ is the tire pneumatic trail. Finally, the driverautomation shared driving model for lane keeping performance is obtained and the state-space model is determined by combing Eqn. (1) Eqn. (4):

$$
\begin{gathered}
\dot{x}(t)=A \mathbf{x}(t)+B_{1}\left(T_{a}+T_{d}\right)+B_{2} w(t), \\
A=\left[\begin{array}{cccccc}
a_{11} & a_{12} & 0 & 0 & a_{15} & 0 \\
a_{21} & a_{22} & 0 & 0 & a_{25} & 0 \\
0 & 1 & 0 & 0 & 0 & 0 \\
1 & l_{s} & v_{x} & 0 & 0 & 0 \\
0 & 0 & 0 & 0 & 0 & 1 \\
a_{61} & a_{62} & 0 & 0 & a_{65} & a_{66}
\end{array}\right], \quad B_{1}=\left[\begin{array}{c}
0 \\
0 \\
0 \\
0 \\
0 \\
b_{11}
\end{array}\right], \\
B_{2}=\left[\begin{array}{llllll}
b_{21} & b_{22} & 0 & 0 & 0 & 0
\end{array}\right]^{\top},
\end{gathered}
$$

where

$$
\begin{aligned}
a_{11} & =-\frac{2\left(C_{r}+C_{f}\right)}{m v_{x}}, & a_{12} & =-v_{x}+\frac{2\left(l_{r} C_{r}-l_{f} C_{f}\right)}{m v_{x}}, \\
a_{15} & =\frac{2 C_{f}}{m}, & a_{21} & =\frac{2\left(l_{r} C_{r}-l_{f} C_{f}\right)}{I_{z} v_{x}}, \\
a_{22} & =-\frac{2\left(l_{r}^{2} C_{r}+l_{f}^{2} C_{f}\right)}{I_{z} v_{x}}, & a_{25} & =\frac{2 l_{f} C_{f}}{I_{z}} \\
a_{61} & =\frac{2 C_{f} n_{R}}{I_{s} R_{s}^{2} v_{x}}, & a_{62} & =\frac{2 C_{f} l_{f} n_{R}}{I_{s} R_{s}^{2} v_{x}} \\
a_{65} & =-\frac{2 C_{f} n_{R}}{I_{s} R_{s}^{2}}, & a_{66} & =\frac{-B_{s}}{I_{s}}, \\
b_{11} & =\frac{1}{I_{s} R_{s}}, & b_{21} & =\frac{1}{m}, \quad b_{22}=\frac{l_{w}}{I_{z}} .
\end{aligned}
$$

In the state-space model, $x=\left[\begin{array}{llllll}v_{y} & r & \psi_{l} & y_{L} & \delta & \dot{\delta}\end{array}\right]^{\top}$ is the state vector. Assistant torque $T_{a}$ and driver torque $T_{d}$ are the system input. $w(t)$ means external disturbance and it shows the lateral wind force here. In this work, an appropriate assistant torque, which can suit the driver's current driving behavior, is desired to be designed. Considering uncertain behavior of human driver, a two-point previewed driver model is introduced as follows: [17]:

$$
T_{d}=K_{d 1} \theta_{\text {near }}+K_{d 2} \theta_{\text {far }} .
$$

Here, $\theta_{\text {near }}$ represents visual direction to the near point, which is related to the driver's present activity. $\theta_{\text {far }}$ indicates visual direction to the far point, which is related to the driver's expectant activity. $K_{d 1}$ and $K_{d 2}$ are gains in accordance with the two angles $\theta_{\text {near }}$ and $\theta_{\text {far }}$ respectively, which represent driver's proportional actions on the two angles. The two visual angles $\theta_{\text {near }}$ and $\theta_{\text {far }}$ are defined as:

$$
\begin{aligned}
\theta_{\text {near }} & =\frac{y_{L}}{v_{x} T_{p}}+\psi_{L}, \\
\theta_{\text {far }} & =\tau_{a}^{2} a_{21} v_{y}+\left(\tau_{a}+\tau_{a}^{2} a_{22}\right) r+\tau_{a}^{2} a_{25} R_{s},
\end{aligned}
$$

where $T_{p}$ is the preview time and $\tau_{a}$ is the react time of human driver. Then, the predicted driver torque can be rewritten as follows:

$$
T_{d}=\left[\begin{array}{llllll}
c_{1} & c_{2} & c_{3} & c_{4} & c_{5} & 0
\end{array}\right] x
$$

where

$$
\begin{array}{ll}
c_{1}=K_{d 2} \tau_{a}^{2} a_{21}, & c_{2}=K_{d 2}\left(\tau_{a}+\tau_{a}^{2} a_{22}\right), \quad c_{3}=K_{d 1}, \\
c_{4}=\frac{K_{d 1}}{v_{x} T_{p}}, & c_{5}=K_{d 2} \tau_{a}^{2} a_{25} R_{s} .
\end{array}
$$

For the driver-automation shared driving system, both the driver torque and the assistant torque are system input. Meanwhile, the pursuit of this work is to design an appropriate assistant torque according to the driver's current behavior. As the driver torque is predicted, the assistant torque could be designed through properly designed controller. It should be emphasized that the designed assistant torque should have ability to be adjusted according to the driver's behavior, to avoid disturbing the human driver.

\section{B. Driver's adaptive need for assistance}

The interaction between human driver and vehicular automation system plays a key role in driver-automation shared control. On the one hand, the human driver must have an adaptive control authority to the vehicle. On the other hand, the assist torque from automation system should be adapt to the driver's real-time activity. When the driver is in distraction, for example, his/her currently driving performance is poor. In this case, the assistant system should provide assistance with high level. Moreover, the assistance level should decrease along with the increase of driver's driving performance, to ensure a comfortable driving experience for the driver. In addition, when the human driver is highly focused on the driving task but he/she is in overload, like the circumstance where human driver is in a dangerous situation, the need for assistance should also be in high level. That is to say, adaptive level of assistance should be provided to undertake the driving tasks [33]. Following these guidelines, the assistant torque from vehicular automation system is modulated as follows:

$$
T_{a}=\mu\left(\theta_{d}(t)\right) u(t), \quad \theta_{d}(t) \in[0,1],
$$

where $u(t)$ is the control input to be designed. The timevarying parameter $\mu\left(\theta_{d}(t)\right)$ is a function of the driver's activity, it shows the driver's need for assistance. Based on it, the assist torque $T_{a}$ can be adapted according to the driver's real-time activity. It should be noted that the driver's activity contains two types of information: 1) the driver's torque; 2) the driver's state. Hence, the detailed description of $\mu\left(\theta_{d}(t)\right)$ is given as follows: [17]:

$$
\begin{aligned}
\mu\left(\theta_{d}(t)\right) & =\omega_{1}\left(\theta_{d}(t)-\omega_{2}\right)^{2}+\mu_{\text {min }}, \\
\theta_{d}(t) & =1-e^{-\left(\sigma_{1} T_{d N}\right)^{\sigma_{2}} D S^{\sigma_{3}},}, \\
T_{d N} & =\left|\frac{T_{d}}{T_{d \max }}\right| .
\end{aligned}
$$

Here, $\theta_{d}(t)$ represents the driver's real-time activity. It could be described by two parameters i.e. $T_{d N}, D S . T_{d N}$ is the driver's normalized torque and it describes the driver's workload. $D S$ $(0 \leq D S \leq 1)$ shows information of driver's state and it describes the driver's attention on the driving task. Note that it means that the driver is divorced from the driving task when $D S=0$, and the driver is highly focused on the driving task when $D S=1$. 
$\omega_{1}, \omega_{2}$ and $\mu_{\min }$ are parameters of the parabolic equation $\mu\left(\theta_{d}(t)\right)$. Thereinto, $\mu_{\min }$ is the minimal assistance level and it could be obtained based on the practical demand. It is worthy mentioning that the minimal assistance level should guarantee the weighting parameter $\mu\left(\theta_{d}\right)$ has a large range. $\sigma_{1}, \sigma_{2}$ and $\sigma_{3}$ are weight parameters of the driver's torque and the driver's state [17].

As the uncertain driver behavior is approximately predicted by Eqn. (8) and the assistance torque is designed through Eqn. (9), substituting Eqn. (8) and Eqn. (9) into Eqn. (5), the driverautomation shared driving system used for controller design purpose can be obtained as follows:

$$
\dot{x}(t)=\bar{A} x(t)+\bar{B}_{1} u(t)+B_{2} w(t),
$$

where

$\bar{A}=\left[\begin{array}{cccccc}a_{11} & a_{12} & 0 & 0 & a_{15} & 0 \\ a_{21} & a_{22} & 0 & 0 & a_{25} & 0 \\ 0 & 1 & 0 & 0 & 0 & 0 \\ 1 & l_{s} & v_{x} & 0 & 0 & 0 \\ 0 & 0 & 0 & 0 & 0 & 1 \\ \bar{a}_{61} & \bar{a}_{62} & \bar{a}_{63} & \bar{a}_{64} & \bar{a}_{65} & a_{66}\end{array}\right], \bar{B}_{1}=\left[\begin{array}{c}0 \\ 0 \\ 0 \\ 0 \\ 0 \\ \bar{b}_{61}\end{array}\right]$

$\bar{a}_{61}=a_{61}+b_{11} c_{1}, \quad \bar{a}_{62}=a_{62}+b_{11} c_{2}, \quad \bar{a}_{65}=a_{65}+b_{11} c_{5}$, $\bar{a}_{63}=b_{11} c_{3}, \quad \bar{a}_{64}=b_{11} c_{4}, \quad \bar{b}_{61}=b_{11} \mu\left(\theta_{d}(t)\right)$.

For a control system, the control output could be defined based on the performance demand. As the lane keeping performance is studied in this work, the tracking performance should be guaranteed first. Apart from it, as the shared control strategy is proposed to reduce conflict between human driver and vehicular automation, the difference between them should also be considered. Besides, when there exists assistance for human driver, considering the road sense, the steering angle should not change too quickly, which may cause discomfort for the human driver. Therefore, its derivative is also an important factor to evaluate the proposed method. Consequently, the control output in this work is defined as:

$$
\begin{aligned}
z(t)= & {\left[\begin{array}{lccccc}
a_{y} & \theta_{\text {near }} & \theta_{\text {far }} & \dot{\delta} & T_{d}-T_{a}
\end{array}\right], } \\
= & E x(t)+F u(t), \\
E & {\left[\begin{array}{cccccc}
0 & v_{x} & 0 & 0 & 0 & 0 \\
0 & 0 & 1 & \frac{1}{v_{x} T_{p}} & 0 & 0 \\
\tau_{a}^{2} a_{21} & \tau_{a}+\tau_{a}^{2} a_{22} & 0 & 0 & \tau_{a}^{2} a_{23} & 0 \\
0 & 0 & 0 & 0 & 0 & 1 \\
c_{1} & c_{2} & c_{3} & c_{4} & c_{5} & 0
\end{array}\right], } \\
F & =\left[\begin{array}{lllll}
0 ; & 0 ; & 0 ; & 0 ; & \mu\left(\theta_{d}(t)\right)
\end{array}\right] .
\end{aligned}
$$

Remark 1. It should be stressed that the vehicle longitudinal speed $v_{x}(t)$ and the driver activity variable $\theta_{d}(t)$ involved in system (11) are time-varying. Moreover, measurement error of $v_{x}(t)$ is inevitable in engineering and the parameter $D S$ in $\theta_{d}(t)$ is also changed. Therefore, the type-1 T-S fuzzy technology depending on a precise vehicle speed in [17] may not hold for the practical application.

Remark 2. In this work, a shared control strategy for the lane keeping assistance system is studied, therefore, both of the tracking performance and the conflict between human driver and automation system should be considered. Although a time-varying parameter $\mu\left(\theta_{d}(t)\right)$ is introduced to adjust the assistant torque according to human driver's activity, a global optimization index aiming to reduce the discrepancy between human driver and automation system is also necessary. Different from the work in [17], a variant control output considering difference between the driver's torque and the assistant torque is introduced, to attenuate conflict between them.

\section{Driver-Automation Shared Control Design}

This section illustrates the type-2 fuzzy driver-automation shared driving model and the corresponding control strategy.

\section{A. Type-2 Fuzzy Driver-Automation Shared Driving Model}

In the driver-automation shared driving system (11), the vehicle longitudinal velocity is time-varying, which leads to a linear parameter-varying system. As well known, conventional type-1 T-S fuzzy model is valid to describe such system and then the membership function could be obtained based on the characteristic of the time-varying parameter. However, as mentioned above, there is inevitable error in the vehicle longitudinal speed. It will cause uncertainty in the membership function, which cannot be addressed by the type-1 fuzzy theory. The uncertain membership function could degrade the performance of designed driver-automation shared controller. To ensure better control performance, the uncertainty in the membership function resulted from measurement error must be addressed. Differing from the existing work in which the vehicle longitudinal speed $v_{x}$ is assumed to be exactly known, the type-2 fuzzy technology is introduced here, to further solve the uncertainty in the membership function. Apart from the vehicle speed, the weighting function $\mu\left(\theta_{d}(t)\right)$ is also timevariant within its limitation, which shows the driver's need for assistance. Furthermore, it is related to the driver's state that can be described by the parameter $D S$. $D S$ is also timevariant and changes in the range of its limitation, which could also cause uncertainty in the membership function.

All of these explain the reason why a new type- 2 fuzzy model is applied here to describe the driver-automation shared driving system (11), which is expressed as follows:

$$
\begin{gathered}
\text { Rule } i: \quad \text { If } f_{1}(t) \text { is } M_{i}^{1} \text { and } \cdots \text { and } f_{p}(t) \text { is } M_{i}^{p} \\
\text { THEN } \dot{x}(t)=\bar{A}_{i} x(t)+\bar{B}_{1 i} u(t)+B_{2 i} w(t), \\
z(t)=C_{i} x(t)
\end{gathered}
$$

where $p$ is the number of premise variable. $M_{i}^{s}$ is the interval type-2 fuzzy set of function $f_{s}(t)$ based on rule $i$, with $i \in\{1,2, \ldots, r\}$ and $s \in\{1,2, \ldots, p\}$. The local state-space matrices $\bar{A}_{i} \in R^{n \times n}, \bar{B}_{1 i} \in R^{n \times m}, B_{2 i}$ and $C_{i}$ are known. The interval sets in accordance with firing strength of the $i$ th rule are shown as:

$$
\begin{aligned}
\widetilde{\xi}_{i}(t) & =\left[\xi_{i}^{L}(t), \xi_{i}^{U}(t)\right], \quad i=1,2, \ldots, r \\
\xi_{i}^{L}(t) & =\underline{\alpha}_{M_{i}^{1}}\left(f_{1}(t)\right) \times \cdots \times \underline{\alpha}_{M_{i}^{p}}\left(f_{p}(t)\right)=\prod_{s=1}^{p} \underline{\alpha}_{M_{i}^{s}}\left(f_{s}(t)\right), \\
\xi_{i}^{U}(t) & =\bar{\alpha}_{M_{i}^{1}}\left(f_{1}(t)\right) \times \cdots \times \bar{\alpha}_{M_{i}^{p}}\left(f_{p}(t)\right)=\prod_{s=1}^{p} \bar{\alpha}_{M_{i}^{s}}\left(f_{s}(t)\right),
\end{aligned}
$$


where $\underline{\alpha}_{M^{s}}\left(f_{s}(t)\right)$ and $\bar{\alpha}_{M_{i}^{s}}\left(f_{s}(t)\right)$ represent the lower and upper grades of membership governed by the lower and upper membership functions, respectively. Note that

$$
0 \leq \underline{\alpha}_{M_{i}^{s}}\left(f_{s}(t)\right) \leq \alpha_{M_{i}^{s}}\left(f_{s}(t)\right) \leq \bar{\alpha}_{M_{i}^{s}}\left(f_{s}(t)\right) \leq 1 .
$$

Then, the interval type-2 fuzzy model of driver-automation shared driving system (11) is defined as

$$
\begin{aligned}
& \dot{x}(t)=\sum_{i=1}^{r} \xi_{i}(t)\left(\bar{A}_{i} x(t)+\bar{B}_{1 i} u(t)+B_{2 i} w(t)\right), \\
& z(t)=\sum_{i=1}^{r} \xi_{i}(t) C_{i} x(t),
\end{aligned}
$$

where

$$
\begin{aligned}
& \xi_{i}(t)=\xi_{i}^{L}(t) \underline{\nu}_{i}(t)+\xi_{i}^{U}(t) \bar{\nu}_{i}(t), \quad \sum_{i=1}^{r} \xi_{i}(t)=1, \\
& 0 \leq \underline{\nu}_{i}(t) \leq 1, \quad 0 \leq \bar{\nu}_{i}(t) \leq 1, \quad \underline{\nu}_{i}(t)+\bar{\nu}_{i}(t)=1 .
\end{aligned}
$$

Here, $\underline{\nu}_{i}(t)$ and $\bar{\nu}_{i}(t)$ are nonlinear function. A practical assumption on longitudinal speed is that $v_{x}(t) \in\left[v_{\min }, v_{\max }\right]$, where $v_{\min }=2.5 \mathrm{~m} / \mathrm{s}$ and $v_{\max }=25 \mathrm{~m} / \mathrm{s}$. Meanwhile, the weighting function $\mu\left(\theta_{d}(t)\right)$ is time-varying with the property of $\mu\left(\theta_{d}(t)\right) \in\left[\mu_{\min }, \mu_{\max }\right]$. There are three premise variables $v_{x}(t), \frac{1}{v_{x}(t)}$ and $\mu\left(\theta_{d}(t)\right)$ involved in system. Note that the number of fuzzy subsystems increases exponentially according to increase on the number of premise variables. Then, the workload on computation will be increased. Here, in order to reduce the computation complexity, a new premise variable $v$ is introduced to decouple $v_{x}(t)$ and $\frac{1}{v_{x}(t)}$, which is shown as follows [17]:

$$
\begin{aligned}
\frac{1}{v_{x}(t)} & =\frac{v_{0}-v_{1}}{v_{1} v_{0}} v+\frac{1}{v_{0}}, & v_{x}(t) & =\left(v_{1}-v_{0}\right) v+v_{0}, \\
v_{0} & =v_{\min }, & v_{1} & =v_{\max },
\end{aligned}
$$

where $v \in[0,1]$. It should be noted that the new premise variable is imprecise as the speed $v_{x}(t)$ is imprecise. Then, the number of premise variable is reduced and the new two premise variables $v, \mu\left(\theta_{d}(t)\right)$ can be expressed as:

$$
f_{1}=v \in\left[f_{1 \min }, f_{1 \max }\right], \quad f_{2}=\mu\left(\theta_{d}(t)\right) \in\left[f_{2 \min }, f_{2 \max }\right] .
$$

Since the number of premise variables is reduced from 3 to 2 , the complexity of driver-automation shared driving system is significantly released. In addition, the error of $v_{x}(t)$ is assumed to be within $\pm 10 \%$, based on which the lower and upper membership functions can be referred in Table I.

\section{B. Type-2 Fuzzy Controller Design}

As the type-2 fuzzy driver-automation shared driving model is defined, the following type-2 fuzzy shared controller is applied to stabilize the system (16):

$$
\text { Rule } j: \quad \text { IF } f_{1}(t) \text { is } M_{j}^{1} \text { and } \cdots \text { and } f_{p}(t) \text { is } M_{j}^{p}
$$$$
\text { THEN } u(t)=G_{j} x(t) \text {, }
$$

where $G_{j}, j \in\{1, \ldots, r\}$ are the state-feedback gains to be designed. Then, the corresponding output of the interval type-2 fuzzy controller is defined as:

$$
u(t)=\sum_{j=1}^{r}\left(\underline{\xi}_{j}(t)+\bar{\xi}_{j}(t)\right) G_{j} x(t),
$$

where

$$
\begin{aligned}
\underline{\xi}_{j}(t) & =\frac{\xi_{j}^{L}(t)}{\sum_{l=1}^{r}\left(\xi_{l}^{L}(t)+\xi_{l}^{U}(t)\right)}, \\
\bar{\xi}_{j}(t) & =\frac{\xi_{j}^{U}(t)}{\sum_{l=1}^{r}\left(\xi_{l}^{L}(t)+\xi_{l}^{U}(t)\right)} .
\end{aligned}
$$

With the expressions in (16) and (20), the closed-loop type-2 fuzzy driver-automation shared control system can be obtained as:

$$
\begin{aligned}
\dot{x}(t) & =\sum_{i=1}^{r} \sum_{j=1}^{r} \xi_{i}\left(\underline{\xi}_{j}+\bar{\xi}_{j}\right)\left[\left(\bar{A}_{i}+\bar{B}_{1 i} G_{j}\right) x(t)+B_{2 i} w(t)\right], \\
z(t) & =\sum_{i=1}^{r} \xi_{i}\left[E_{i} x(t)+F_{i} u(t)\right], \\
& =\sum_{i=1}^{r} \sum_{j=1}^{r} \xi_{i}\left(\underline{\xi}_{j}+\bar{\xi}_{j}\right)\left[\left(E_{i}+F_{i} G_{j}\right) x(t)\right] .
\end{aligned}
$$

For simplicity, $\xi_{i}(t), \underline{\xi}_{j}(t)$ and $\bar{\xi}_{j}(t)$ are replaced with $\xi_{i}$, $\underline{\xi}_{j}$ and $\bar{\xi}_{j}$, respectively. Besides, they have the following characteristic:

$$
\sum_{i=1}^{r} \xi_{i}=\sum_{j=1}^{r}\left(\underline{\xi}_{j}+\bar{\xi}_{j}\right)=\sum_{i=1}^{r} \sum_{j=1}^{r} \xi_{i}\left(\underline{\xi}_{j}+\bar{\xi}_{j}\right)=1 .
$$

Then, the driver-automation shared controller design is transformed to select matrices $G_{j}$ for the closed-loop system (21). However, when pursuing the control effect, the robustness should also be considered, which means the system itself should have ability to attenuate the influence of external disturbance on the control output $z(t)$. An $\mathcal{H}_{\infty}$ control method, therefore, is employed to evaluate the influence mentioned before, which is shown as:

$$
\|z\|_{2}<\gamma\|w\|_{2} .
$$

In addition, in order to obtain better transient performance, a $\mathcal{D}$-stability method is applied in this work. As well known, the transient performance of control system could be improved with its eigenvalues faring away from the imaginary axis, with the cost of huge control input. Fortunately, $\mathcal{D}$-stability is a valid method to realize it without enlarging effort of the control system.

Lemma 1. [34] $\mathcal{D}$-stability: A real matrix $\bar{A}$ is $\mathcal{D}$-stable, i.e. all the eigenvalues of the matrix $\bar{A}$ are in the LMI region $\mathcal{D}$, if and only if there exists a positive matrix $P=P^{\top}$ such that

$$
\mathcal{M}_{\mathcal{D}}(\bar{A}, P)=\Gamma \bigotimes P+\Upsilon \bigotimes(X \bar{A})+\Upsilon^{\top} \bigotimes\left(\bar{A}^{\top} X\right)<0 .
$$

Specially, for a disk LMI region $(q, r)$, the condition for the $\mathcal{D}$-stability is

$$
\left[\begin{array}{cc}
-r P & q P+P \bar{A} \\
* & -r P
\end{array}\right] \leq 0 .
$$


TABLE I

TYPE-2 FUZZY MEMBERSHIP FUNCTIONS

\begin{tabular}{|c|c|c|c|}
\hline \multicolumn{2}{|c|}{ Lower and upper membership functions } \\
\hline \multicolumn{2}{|c|}{$f_{1}$} & \multicolumn{2}{|c|}{$f_{2}$} \\
\hline \multirow{2}{*}{$\Delta=-10 \%$} & $\bar{\alpha}_{M_{1}^{1}}=\bar{\alpha}_{M_{2}^{1}}=\frac{f_{1 \max }-0.9 f_{1}}{f_{1 \max }-f_{1 \min }}$ & \multirow{2}{*}{$D S=0.5$} & $\underline{\alpha}_{M_{1}^{2}}=\underline{\alpha}_{M_{3}^{2}}=\frac{f_{2 \max }-f_{2}}{f_{2 \max }-f_{2 \min }}$ \\
& $\underline{\alpha}_{M_{3}^{1}}=\underline{\alpha}_{M_{4}^{1}}=\frac{0.9 f_{1}-f_{1 \min }}{f_{1 \max }-f_{1 \min }}$ & & $\underline{\alpha}_{M_{2}^{2}}=\underline{\alpha}_{M_{4}^{2}}=\frac{f_{2}-f_{2 \min }}{f_{2 \max }-f_{2 \min }}$ \\
\hline \multirow{3}{*}{$\Delta=10 \%$} & $\underline{\alpha}_{M_{1}^{1}}=\underline{\alpha}_{M_{2}^{1}}=\frac{f_{1 \max }-1.1 f_{1}}{f_{1 \max }-f_{1 \min }}$ & \multirow{5}{*}{$D S=1$} & $\bar{\alpha}_{M_{1}^{2}}=\bar{\alpha}_{M_{3}^{2}}=\frac{f_{2 \max }-f_{2}}{f_{2 \max }-f_{2 \min }}$ \\
\cline { 2 - 2 } & $\bar{\alpha}_{M_{3}^{1}}=\bar{\alpha}_{M_{4}^{1}}=\frac{1.1 f_{1}-f_{1 \min }}{f_{1 \max }-f_{1 \min }}$ & & $\bar{\alpha}_{M_{2}^{2}}=\bar{\alpha}_{M_{4}^{2}}=\frac{f_{2}-f_{2 \min }}{f_{2 \max }-f_{2 \min }}$ \\
\hline
\end{tabular}

$$
\left[\begin{array}{ccc}
L_{j 1}+L_{j 1}^{\top}+N_{i 1}+N_{i 1}^{\top}-V_{i j 1}-V_{i j 1}^{\top}+Q_{i j} & B_{2 i}+L_{j 2}+L_{j 3}^{\top}+N_{i 2}+N_{i 3}^{\top}-V_{i j 2}-V_{i j 3}^{\top} & \left(E_{i} X+F_{i} N_{j}\right)^{\top} \\
* & -\gamma^{2} I+L_{j 4}+L_{j 4}^{\top}+N_{i 4}+N_{i 4}^{\top}-V_{i j 4}-V_{i j 4}^{\top} & 0 \\
* & * & -\frac{1}{2} I
\end{array}\right] \leq 0,
$$

$$
\left[\begin{array}{ccc}
M_{j 1}+M_{j 1}^{\top}+N_{i 1}+N_{i 1}^{\top}-U_{i j 1}-U_{i j 1}^{\top}+Q_{i j} & B_{2 i}+M_{j 2}+M_{j 3}^{\top}+N_{i 2}+N_{i 3}^{\top}-U_{i j 2}-U_{i j 3}^{\top} & \left(E_{i} X+F_{i} N_{j}\right)^{\top} \\
* & -\gamma^{2} I+M_{j 4}+M_{j 4}^{\top}+N_{i 4}+N_{i 4}^{\top}-U_{i j 4}-U_{i j 4}^{\top} & 0 \\
* & * & -\frac{1}{2} I
\end{array}\right] \leq 0,
$$

C. Stability Analysis of Type-2 Fuzzy Shared Control System

The following theorem provides sufficient conditions for designing the type-2 fuzzy driver-automation shared controller with a promised $\mathcal{H}_{\infty}$ performance level.

Theorem 1. Given a positive scalar $\gamma$, the closed-loop type-2 fuzzy control system (21) is asymptotically and robustly stable, the $\mathcal{H}_{\infty}$ condition in (23) is satisfied and the $\mathcal{D}$-stability in a disk LMI region $(q, r)$ is ensured if there exist matrices of appropriate dimensions $X=X^{\top}, K_{j}=G_{j} X, L_{j 1}, M_{j 1}, N_{j 1}$, $L_{j 2}, M_{j 2}, N_{j 2}, L_{j 3}, M_{j 3}, N_{j 3}, L_{j 4}, M_{j 4}, N_{j 4}, R_{j j 1}=R_{j j 1}^{\dagger}$, $S_{j j 1}=S_{j j 1}^{\top}, T_{i j 1}=T_{i j 1}^{\top}, U_{i j 1}, V_{i j 1}, W_{i j 1}, R_{j j 2}=R_{j j 2}^{\top}$, $S_{j j 2}=S_{j j 2}^{\top}, T_{j j 2}=T_{j j 2}^{\top}, U_{i j 4}, V_{i j 4}, W_{i j 4}, U_{i j 2}, V_{i j 2}, W_{i j 2}$, $U_{i j 3}, V_{i j 3}$ and $W_{i j 3}$ such that

$$
\begin{aligned}
& {\left[\begin{array}{cc}
R_{j j 1} & 0 \\
0 & R_{j j 2}
\end{array}\right] \leq\left[\begin{array}{cc}
N_{j 1}+N_{j 1}^{\top} & N_{j 2}+N_{j 3}^{\top} \\
* & N_{j 4}+N_{j 4}^{\top}
\end{array}\right],} \\
& {\left[\begin{array}{cc}
S_{j j 1} & 0 \\
0 & S_{j j 2}
\end{array}\right] \leq\left[\begin{array}{cc}
L_{j 1}+L_{j 1}^{\top} & L_{j 2}+L_{j 3}^{\top} \\
* & L_{j 4}+L_{j 4}^{\top}
\end{array}\right]} \\
& {\left[\begin{array}{cc}
T_{j j 1} & 0 \\
0 & T_{j j 2}
\end{array}\right] \leq\left[\begin{array}{cc}
M_{j 1}+M_{j 1}^{\top} & M_{j 2}+M_{j 3}^{\top} \\
* & M_{j 4}+M_{j 4}^{\top}
\end{array}\right]} \\
& {\left[\begin{array}{cc}
W_{i j 1}+W_{i j 1}^{\top} & W_{i j 2}+W_{i j 3}^{\top} \\
* & W_{i j 4}+W_{i j 4}^{\dagger}
\end{array}\right] \leq} \\
& {\left[\begin{array}{cc}
M_{j 1}+M_{j 1}^{\top}+L_{i 1}+L_{i 1}^{\top} & M_{j 2}+M_{j 3}^{\top}+L_{i 2}+L_{i 3}^{\top} \\
* & M_{j 4}+M_{j 4}^{\top}+L_{i 4}+L_{i 4}^{\top}
\end{array}\right],} \\
& {\left[\begin{array}{ccc}
-R & V & U \\
* & -S & -W \\
* & * & -T
\end{array}\right]<0,}
\end{aligned}
$$

and

$$
\left[\begin{array}{cc}
-r X & q X+\bar{A}_{i} X+\bar{B}_{1 i} N_{j} \\
* & -r X
\end{array}\right] \leq 0 .
$$

Here,

$$
\begin{aligned}
& V=\left[\begin{array}{ccc}
V_{11} & \cdots & V_{1 r} \\
\vdots & \ddots & \vdots \\
V_{r 1} & \cdots & V_{1 r}
\end{array}\right]^{\top}, \quad U=\left[\begin{array}{ccc}
U_{11} & \cdots & U_{1 r} \\
\vdots & \ddots & \vdots \\
U_{r 1} & \cdots & U_{1 r}
\end{array}\right]^{\top}, \\
& W=\left[\begin{array}{ccc}
W_{11} & \cdots & W_{1 r} \\
\vdots & \ddots & \vdots \\
W_{r 1} & \cdots & W_{1 r}
\end{array}\right]^{\top}, \quad R=\left[\begin{array}{ccc}
R_{11} & 0 & 0 \\
0 & \ddots & 0 \\
0 & 0 & R_{r r}
\end{array}\right], \\
& S=\left[\begin{array}{ccc}
S_{11} & 0 & 0 \\
0 & \ddots & 0 \\
0 & 0 & S_{r r}
\end{array}\right], \quad T=\left[\begin{array}{ccc}
T_{11} & 0 & 0 \\
0 & \ddots & 0 \\
0 & 0 & T_{r r}
\end{array}\right], \\
& U_{i j}=\left[\begin{array}{ll}
U_{i j 1} & U_{i j 2} \\
U_{i j 3} & U_{i j 4}
\end{array}\right], \quad V_{i j}=\left[\begin{array}{ll}
V_{i j 1} & V_{i j 2} \\
V_{i j 3} & V_{i j 4}
\end{array}\right], \\
& W_{i j}=\left[\begin{array}{ll}
W_{i j 1} & W_{i j 2} \\
W_{i j 3} & W_{i j 4}
\end{array}\right], \quad \quad R_{j j}=\left[\begin{array}{cc}
R_{j j 1} & 0 \\
0 & R_{j j 2}
\end{array}\right] \text {, } \\
& S_{j j}=\left[\begin{array}{cc}
S_{j j 1} & 0 \\
0 & S_{j j 2}
\end{array}\right], \quad T_{j j}=\left[\begin{array}{cc}
T_{j j 1} & 0 \\
0 & T_{j j 2}
\end{array}\right], \\
& L_{j}=\left[\begin{array}{ll}
L_{j 1} & L_{j 2} \\
L_{j 3} & L_{j 4}
\end{array}\right], \quad \quad M_{j}=\left[\begin{array}{ll}
M_{j 1} & M_{j 2} \\
M_{j 3} & M_{j 4}
\end{array}\right], \\
& N_{j}=\left[\begin{array}{llll}
N_{j 1} & N_{j 2} ; & N_{j 3} & N_{j 4}
\end{array}\right] .
\end{aligned}
$$

When the inequalities in (26) (33) are satisfied, the statefeedback gains can be obtained as follows:

$$
G_{j}=K_{j} X^{-1}, \quad j=1,2, \ldots, r .
$$


Proof. Considering a candidate Lyapunov function presented as follows:

$$
V(t)=x(t)^{\top} P x(t), \quad P=P^{\top}>0 .
$$

Based on Eqn. (21), the time-derivative of candidate Lyapunov function (35) is expressed as

$$
\begin{aligned}
\dot{V}(x(t)) & =\dot{x}(t)^{\top} P x(t)+x(t)^{\top} P \dot{x}(t), \\
& =\sum_{i=1}^{r} \sum_{j=1}^{r} \xi_{i}\left(\underline{\xi}_{j}+\bar{\xi}_{j}\right)\left[\varepsilon(t)^{\top} H e\left(\bar{A}_{i} X+\bar{B}_{1 i} K_{j}\right) \varepsilon(t)\right. \\
& \left.+w(t)^{\top} B_{2 i} \varepsilon(t)+\varepsilon(t)^{\top} B_{2 i} w(t)\right] .
\end{aligned}
$$

Moreover, one has

$$
\begin{array}{rl}
z(t)^{\top} z(t)= & {\left[\sum_{i=1}^{r} \sum_{j=1}^{r} \xi_{i}\left(\underline{\xi}_{j}+\bar{\xi}_{j}\right)\left(E_{i}+F_{i} G_{j}\right) x(t)\right]^{\top}} \\
& {\left[\sum_{l=1}^{r} \sum_{m=1}^{r} \xi_{i}\left(\underline{\xi}_{m}+\bar{\xi}_{m}\right)\left(E_{l}+F_{l} G_{m}\right) x(t)\right],} \\
\leq 2 & 2 \sum_{i=1}^{r} \sum_{j=1}^{r} \xi_{i}\left(\underline{\xi}_{j}+\bar{\xi}_{j}\right)\left[\varepsilon(t)^{\top}\left(E_{i} X+F_{i} N_{j}\right)^{\top}\right. \\
& \left.\left(E_{i} X+F_{i} N_{j}\right) \varepsilon(t)\right],
\end{array}
$$

where $\varepsilon(t)=P x(t), X=P^{-1}, K_{j}=G_{j} X$. Besides, defining that $Q_{i j}=\operatorname{He}\left(\bar{A}_{i} X+\bar{B}_{1 i} K_{j}\right)$ for simplicity. It can be seen that the type-2 fuzzy control system (21) is asymptotically stable if there is $\dot{V}(x(t))<0$. Apart from the stability, the robustness of control system is necessary to be studied as mentioned before, then, an index is defined as:

$$
\begin{aligned}
J & =\dot{V}(x(t))+z(t)^{\top} z(t)-\gamma^{2} w(t)^{\top} w(t), \\
& \leq \sum_{i=1}^{r} \sum_{j=1}^{r} \xi_{i}\left(\underline{\xi}_{j}+\bar{\xi}_{j}\right) \epsilon(t)^{\top} \Theta_{i j} \epsilon(t),
\end{aligned}
$$

where $\epsilon(t)=\left[\varepsilon(t)^{\top} w(t)^{\top}\right]^{\top}, \Theta_{i j}=\left[Q_{i j}+2\left(E_{i} X+\right.\right.$ $\left.\left.F_{i} N_{j}\right)^{\mathrm{T}} I\left(E_{i} X+F_{i} N_{j}\right) \quad B_{2 i} ; B_{2 i}^{\mathrm{T}}-\gamma^{2} I\right]$. It can be seen from (38) that the condition $\Theta_{i j}<0$ can ensure $J<0$. Then, the robustness of control system can be guaranteed. In order to reduce the conservativeness, some slack matrices are introduced here. Hence, a new function is defined as:

$$
\begin{aligned}
\Phi & =\sum_{i=1}^{r} \sum_{j=1}^{r}\left(\xi_{i}-\underline{\xi}_{i}-\bar{\xi}_{i}\right)\left\{\underline{\xi}_{j}\left(L_{j}+L_{j}^{\top}\right)+\bar{\xi}_{j}\left(M_{j}+M_{j}^{\top}\right)\right. \\
& \left.-\xi_{j}\left(N_{j}+N_{j}^{\top}\right)\right\}, \\
& =\sum_{i=1}^{r} \sum_{j=1}^{r}\left\{\xi_{i} \underline{\xi}_{j}\left(L_{j}+L_{j}^{\top}+N_{i}+N_{i}^{\top}\right)\right. \\
& +\xi_{i} \bar{\xi}_{j}\left(M_{j}+M_{j}^{\top}+N_{i}+N_{i}^{\top}\right) \\
& -\xi_{i} \xi_{j}\left(N_{j}+N_{j}^{\top}\right)-\underline{\xi}_{i} \xi_{j}\left(L_{j}+L_{j}^{\top}\right) \\
& \left.-\underline{\xi}_{i} \bar{\xi}_{j}\left(M_{j}+M_{j}^{\top}+L_{i}+L_{i}^{\top}\right)-\bar{\xi}_{i} \bar{\xi}_{j}\left(M_{j}+M_{j}^{\top}\right)\right\},
\end{aligned}
$$

where $L_{j}, M_{j}$ and $N_{j}$ are slack matrices with appropriate dimensions. Note that $\xi_{i}$ has the property of $\sum_{i=1}^{r} \xi_{i}=$ $\sum_{j=1}^{r}\left(\underline{\xi}_{j}+\bar{\xi}_{j}\right)=1, \sum_{i=1}^{r}\left(\xi_{i}-\underline{\xi}_{i}-\bar{\xi}_{i}\right)=0$, therefore, there is $\Phi=0$. By using $\Psi$ to present $\sum_{i=1}^{r} \sum_{j=1}^{r} \xi_{i}\left(\underline{\xi}_{j}+\bar{\xi}_{j}\right) \Theta_{i j}$ in Eqn. (38), the following expression is obtained:

$$
\begin{aligned}
\Phi+\Psi & =\sum_{i=1}^{r} \sum_{j=1}^{r}\left\{\xi_{i} \underline{\xi}_{j}\left(L_{j}+L_{j}^{\mathrm{T}}+N_{i}+N_{i}^{\mathrm{T}}+\Theta_{i j}\right)\right. \\
& +\xi_{i} \bar{\xi}_{j}\left(M_{j}+M_{j}^{\mathrm{T}}+N_{i}+N_{i}^{\mathrm{T}}+\Theta_{i j}\right) \\
& -\xi_{i} \xi_{j}\left(N_{j}+N_{j}^{\mathrm{T}}\right)-\underline{\xi}_{i} \underline{\xi}_{j}\left(L_{j}+L_{j}^{\mathrm{T}}\right) \\
& \left.-\underline{\xi}_{i} \bar{\xi}_{j}\left(M_{j}+M_{j}^{\mathrm{T}}+L_{i}+L_{i}^{\mathrm{T}}\right)-\bar{\xi}_{i} \bar{\xi}_{j}\left(M_{j}+M_{j}^{\mathrm{T}}\right)\right\} .
\end{aligned}
$$

By applying Schur complement to conditions in (26) and (27) respectively, the two following equalities can be obtained:

$$
\begin{gathered}
L_{j}+L_{j}^{\mathrm{T}}+N_{i}+N_{i}^{\mathrm{T}}+\Theta_{i j} \leq V_{i j}+V_{i j}^{\mathrm{T}}, \\
M_{j}+M_{j}^{\mathrm{T}}+N_{i}+N_{i}^{\mathrm{T}}+\Theta_{i j} \leq U_{i j}+U_{i j}^{\mathrm{T}} .
\end{gathered}
$$

With conditions in $(41) \sim(42)$ and $(28) \sim(31)$, the condition in (40) can be transformed to

$$
\begin{aligned}
& \Phi+\Psi \leq \sum_{i=1}^{r} \sum_{j=1}^{r}\left[\xi_{i} \underline{\xi}_{j}\left(V_{i j}+V_{i j}^{\top}\right)+\xi_{i} \bar{\xi}_{j}\left(U_{i j}+U_{i j}^{\top}\right)\right. \\
& \left.-\xi_{i} \xi_{j} R_{j j}-\underline{\xi}_{i} \xi_{j} S_{j j}-\underline{\xi}_{i} \bar{\xi}_{j}\left(W_{i j}+W_{i j}^{\top}\right)-\bar{\xi}_{i} \bar{\xi}_{j} T_{j j}\right] .
\end{aligned}
$$

Then, the index $J$ can be rewritten as

$$
\begin{aligned}
J & =\epsilon(t)^{\top} \Psi \epsilon(t)=\epsilon(t)^{\top}[\Psi+\Phi] \epsilon(t), \\
& \leq\left[\begin{array}{c}
r(t) \\
s(t) \\
q(t)
\end{array}\right]^{\top}\left[\begin{array}{ccc}
-R & V & U \\
* & -S & -W \\
* & * & -T
\end{array}\right]\left[\begin{array}{l}
r(t) \\
s(t) \\
q(t)
\end{array}\right],
\end{aligned}
$$

where

$$
\begin{aligned}
r(t) & =\left[\begin{array}{llll}
\xi_{1} \epsilon(t) & \xi_{2} \epsilon(t) & \cdots & \xi_{r} \epsilon(t)
\end{array}\right]^{\top}, \\
s(t) & =\left[\begin{array}{llll}
\xi_{1} \epsilon(t) & \underline{\xi}_{2} \epsilon(t) & \cdots & \underline{\xi}_{r} \epsilon(t)
\end{array}\right]^{\top}, \\
q(t) & =\left[\begin{array}{llll}
\bar{\xi}_{1} \epsilon(t) & \bar{\xi}_{2} \epsilon(t) & \cdots & \bar{\xi}_{r} \epsilon(t)
\end{array}\right]^{\top} .
\end{aligned}
$$

It can be seen from the condition (32) that $J<0$. With similar steps in [35], negative index $J$ indicates that the closed-loop system (21) is stable and the $\mathcal{H}_{\infty}$ performance is guaranteed. Moreover, it should be emphasized that all the eigenvalues of matrices $\bar{A}_{i}$ should be within the disk LMI region $(q, r)$ to ensure desired transient performance. Based on Lemma 1, the following inequality is obtained:

$$
\left[\begin{array}{cc}
-r P & q P+P\left(\bar{A}_{i}+\bar{B}_{1 i} G_{j}\right) \\
* & -r P
\end{array}\right]<0 .
$$

Performing the congruence transformation to (46) with $\operatorname{diag} P^{-1}, P^{-1}$ and $X=P^{-1}$, condition in (33) can be obtained.

It is observed from Theorem 1 that $\mathcal{H}_{\infty}$ performance index $\gamma$ indicates the attenuation level. Through minimizing the value of $\gamma$, better disturbance attenuation performance can be obtained. Then, the following corollary is introduced:

Corollary 1. The minimum $\mathcal{H}_{\infty}$ performance index $\gamma^{*}$ in Theorem 1 can be obtained by solving the following optimization problem:

$$
\begin{gathered}
\gamma^{*}=\min \gamma, \\
\text { s.t. }(26) \sim(33) .
\end{gathered}
$$




\section{Simulation Results}

In this section, simulation and comparison results are provided. The simulation platform structure is depicted in Fig. 2. By computing a sets of linear matrix inequalities in Theorem 1 with the disk LMI region $(100,100)$, the feed-back gains can be obtained and the optimal $\mathcal{H}_{\infty}$ index $\gamma$ is 0.497 . It should be noted that the vehicle parameters used in the research is referred from [17]. Here, the simulation test is set on the condition where humane driver and vehicular automation cooperatively undertake the driving task, with an adaptive level of assistance for the human driver. In order to demonstrate the superiority of proposed type-2 fuzzy driver-automation shared controller, the type-1 T-S fuzzy controller from the work in [17] is introduced as the comparison. In addition, system constraints referred from [36], which represent the "normal driving" zone for safety.

$$
\begin{aligned}
& G_{1}= \\
& {[-802.5,-1551.2,-6239.1,-4802.5,-16746.4,-137.0],} \\
& G_{2}= \\
& {[-806.0,-1556.5,-6252.2,-4818.0,-16786.9,-137.3],} \\
& G_{3}= \\
& {[-802.8,-1551.1,-6239.0,-4802.1,-16746.1,-136.9]} \\
& G_{4}= \\
& {[-803.4,-1551.9,-6241.8,-4805.7,-16756.6,-137.2]}
\end{aligned}
$$

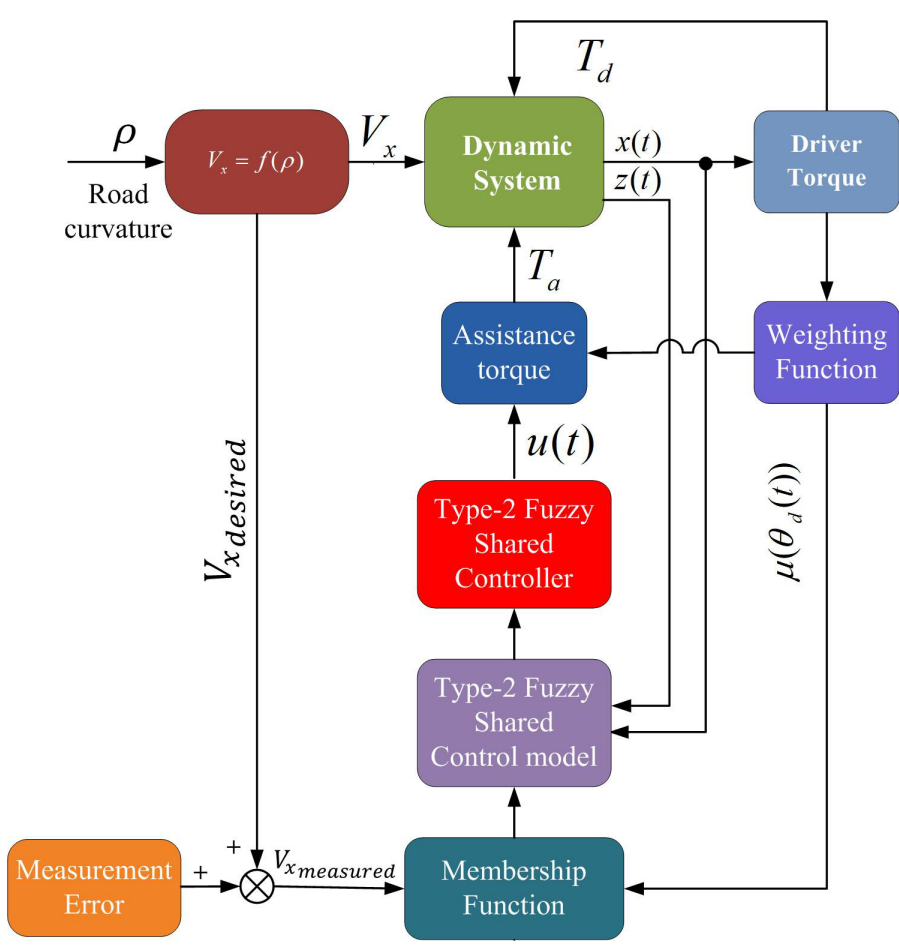

Fig. 2. Simulation schematic diagram.

Note that the proposed type-2 fuzzy controller is designed to ensure better lane keeping performance. In practice, there are many factors such as the road geometry and the traffic flow that have influence on the driving. Both them could affect the vehicle speed and have further effect on the vehicle control. To verify the proposed controller for lane keeping performance, two modes are selected in the simulation experiment. One mode introduces a longitudinal vehicle speed depending on the road curvature and the function between them is adopted from [37]. The other mode relies on the vehicle speed which is measured in the practically urban driving condition, which shows the influence of traffic flow.

In the first simulation test, the provided road curvature is shown in Fig. 3 (a). According to the relationship between them referred in [37], when the vehicle is running on a flat street with good adhesive condition, the calculated vehicle speed is depicted in Fig. 3 (b). It should be noted that there are two types of vehicle speed i.e. desired vehicle speed and measured vehicle speed in the simulation structure. The vehicle speed containing error is sent to the type-2 fuzzy controller module, which could be obtained from through $V_{x_{\text {measured }}}=V_{x_{\text {desired }}}+\Delta V_{x} . \Delta V_{x}$ means the error and it is assumed that there is $\pm 10 \%$ deviation from the sensor, which is a sampled gaussian noise generated by a signal builder. Moreover, the lateral wind force utilized in the simulation test can be referred in Fig. 3 (c).
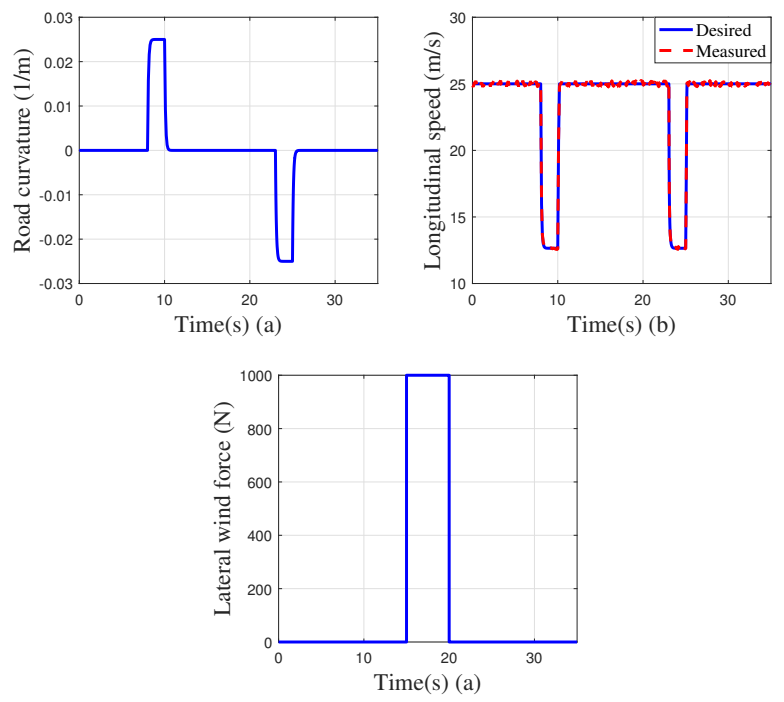

Fig. 3. Given road curvature and the corresponding vehicle speed.

Figs. 4 and 5 depict results of the first simulation test. Note that responses from two different shared controllers are obtained at the same driving pattern. Fig. 4 (a) indicates the vehicle lateral performance and Fig. 4(b) presents the lane keeping performance. As depicted in Figs. 4 (a) and (b), when the lateral wind functions, the proposed type- 2 fuzzy controller brings lesser lateral offset and heading error. It is because the uncertainty resulted from error is considered, otherwise, it will degrade the performance of controller. Fig. 4 (c) shows that the proposed type-2 fuzzy controller provides a smaller overshoot in the response of vehicle yaw rate, which is closely related to the vehicle stability. In contrast, the conventional type-1 fuzzy controller generated a larger overshoot. In addition, large 
overshoot in steering rate should also be avoided, which will cause unexpected anxiety to the human driver. Similarly, it can be seen from Fig. 4 (d) that the proposed type-2 fuzzy controller provides more smaller overshoot than the other. The compared results indicate that the proposed type- 2 controller outdoes the type-1 fuzzy controller.

In order to explain the simulation results in detail, Table II presents comparison results about the root mean squares of vehicle states responding to two kinds of controller, and Table III shows comparison results about the infinite-norm. It can be seen from Table II and Table III that the type-2 fuzzy controller leads to little steady-state error and smaller maximum offset than the type-1 fuzzy controller during the response precess. As a result, it can be concluded that the proposed type- 2 controller is superior than the traditional type1 fuzzy controller.
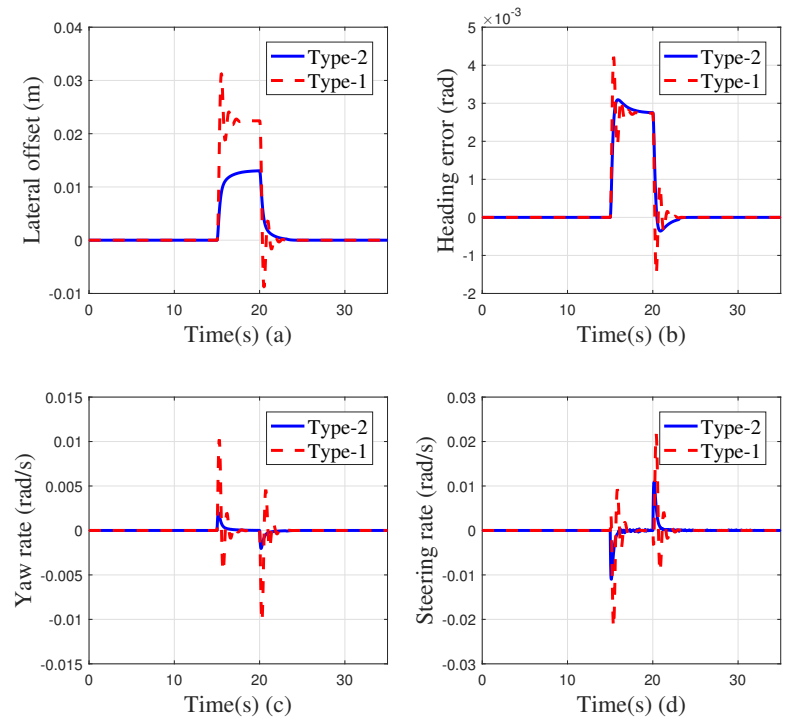

Fig. 4. Response of vehicle states in accordance with given road curvature-I.

Fig. 5 shows information of the assistance level and the torque distribution, which indicates that when the lateral wind occurs, the human driver and the automation have same directional activity and the maximal difference between them is about $3.8 \mathrm{~N} / \mathrm{m}$. Note that the driver torque can be predicted through Eqn. (8). Looking at the information in more detail, Fig. 5 (a) depicts the assistance level in accordance with current driver's behavior. It shows that when there is no external disturbance, the automation can control the vehicle and release human driver from the driving task. When the lateral wind force acts and the human driver detects it, he/she will act to control the steering wheel. As depicted in Fig. 5 (a), the corresponding assistance level decreases and partial control authority is transferred to the human driver at the moment. Note that if there is a biggish external disturbance, support of the automation may remain a high level, it is because the driver could not control the vehicle completely. Therefore, the minimal support is still in a high level in the presence of lateral wind, which is about 0.76 . Similarly, Fig. 5 (b) also shows that the assistance torque is bigger than that of the driver torque
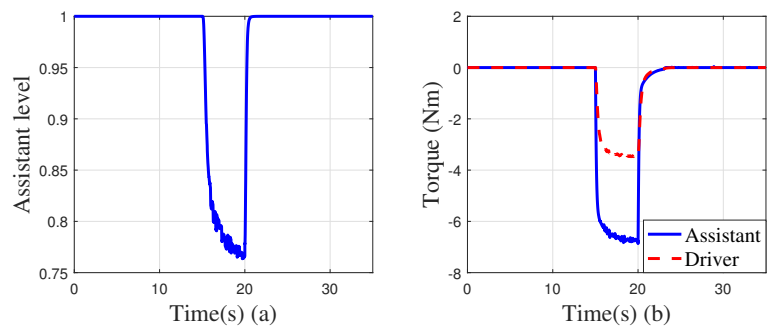

Fig. 5. (a) Assistance level according to driver's activity; (b) The driver torque and the assistance torque.

TABLE II

Root Mean Square (RMS) of Vehicle States Responding to GIVEN ROAD CURVATURE

\begin{tabular}{|c|c|c|}
\hline Vehicle state & Type -1 controller & Type -2 controller \\
\hline Lateral offset & $8.61 e-03$ & $4.60 e-03$ \\
\hline Heading error & $1.06 e-03$ & $1.05 e-03$ \\
\hline Yaw rate & $1.24 e-03$ & $2.60 e-04$ \\
\hline Steering rate & $2.64 e-03$ & $1.29 e-03$ \\
\hline
\end{tabular}

TABLE III

Infinite NoRm of Vehicle States RESPONDING to Given RoAd Curvature

\begin{tabular}{|c|c|c|}
\hline Vehicle state & Type -1 controller & Type -2 controller \\
\hline Lateral offset & $3.13 e-02$ & $1.30 e-02$ \\
\hline Heading error & $4.22 e-03$ & $3.09 e-03$ \\
\hline Yaw rate & $1.02 e-02$ & $2.03 e-03$ \\
\hline Steering rate & $2.17 e-02$ & $1.10 e-02$ \\
\hline
\end{tabular}

In the second simulation test, the measured vehicle speed in urban driving condition is applied, which reflects the influence of urban traffic flow. The experiment data was employed by [38] to demonstrate the effectiveness of an adaptive cruise controller, in which the data were measured from an electric vehicle driven on a urban road (denoted as 'Route A' in the literature) in Cambridge, UK. Figs. 6 and 8 depict results of the second simulation test, thereinto, Fig. 7 (a) depicts the assistance level $\mu\left(\theta_{d}(t)\right)$ according to the driver's behavior and Fig. 7 (b) shows the corresponding torque distribution. Fig. 7 (a) shows that the assistance level decreases when the lateral wind acts, and the procedure is similar to that of the first simulation.

Fig. 8 (a) shows responses of both controllers, which are related to the lateral performance of controlled vehicle. It can be seen from Fig. 8 (a) that the maximal lateral offset of proposed type-2 fuzzy controller is smaller than that of the traditional type- 1 controller. This shows the proposed controller is more precise, as the new type-2 fuzzy technology is applied to address the uncertainty from imprecise speed and changing driver state. Fig. 8 (b) shows the lane keeping performance of both shared controllers with adaptive assistance. Since a smaller heading error indicates better lane keeping 
performance, Fig. 8 (b) indicates the proposed type-2 fuzzy controller could ensure better lane keeping performance, of which the maximal heading error is smaller.

In addition, comparisons on responses of the yaw rate and the steering rate are given in Figs. 8 (c) and (d), respectively. It is well known that the yaw rate is an important index to evaluate the vehicle handling stability and the value is desired to be tiny for the vehicle safety. Furthermore, steering rate can also affect the driver's driving experience, because of the feedback of road sense with his/her hands on the steering wheel. Both of them are important indicators to evaluate the proposed control method. Fortunately, based on comparisons on responses of both controllers, it can be concluded that the proposed type- 2 fuzzy controller is much better than the traditional type- 1 fuzzy controller, as the former provides smaller yaw rate and steering rate. Similarly, compared results from Table IV and Table V also demonstrate the superiority of proposed type-2 fuzzy controller not only in the steady-state error of control system but also in the system overshoot.
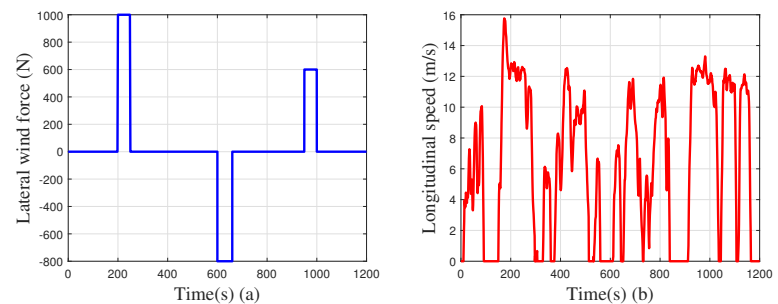

Fig. 6. (a) The external disturbance; (b) The measured vehicle speed.
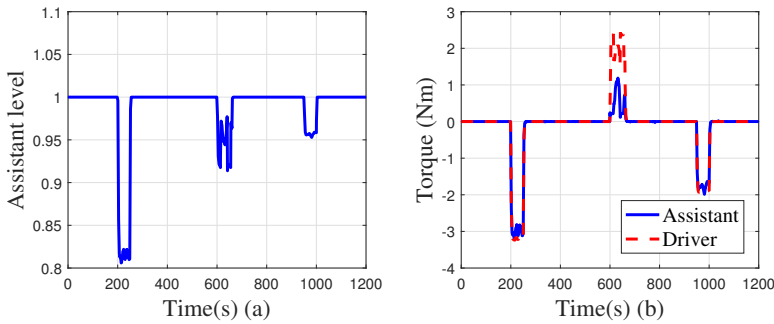

Fig. 7. (a) Assistance level according to driver's activity; (b) The driver torque and the assistance torque.

TABLE IV

Root Mean Square (RMS) of Vehicle States Responding to MEASUREd Vehicle SPEED

\begin{tabular}{|c|c|c|}
\hline Vehicle state & Type -1 controller & Type -2 controller \\
\hline Lateral offset & $6.20 e-03$ & $2.23 e-03$ \\
\hline Heading error & $4.72 e-04$ & $2.91 e-04$ \\
\hline Yaw rate & $1.20 e-03$ & $2.83 e-04$ \\
\hline Steering rate & $1.03 e-04$ & $9,92 e-05$ \\
\hline
\end{tabular}

In this work, the type-2 fuzzy technology is introduced to address imprecise vehicle longitudinal velocity, which can lead to uncertainty in the membership function. In fact, apart form the imprecise measurement of vehicle speed, other uncertain
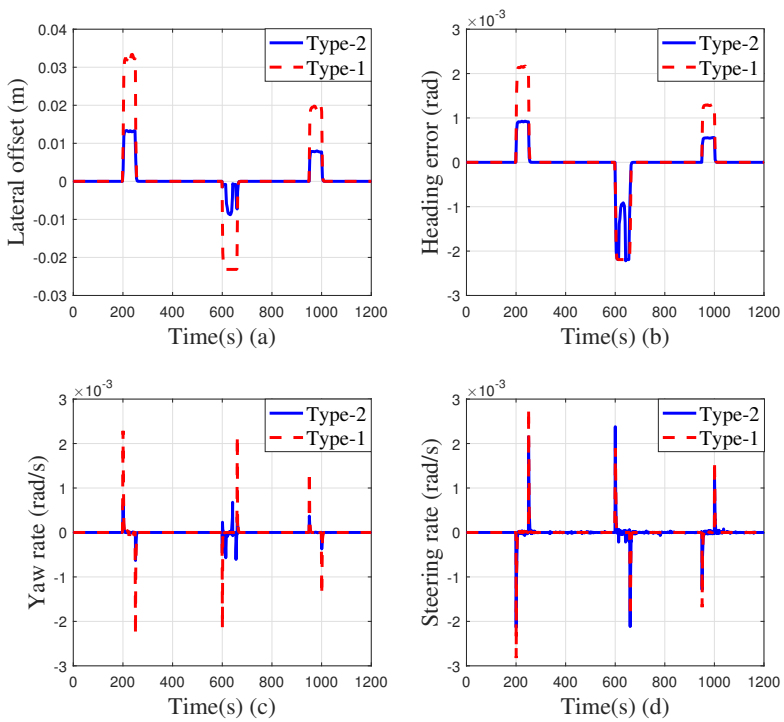

Fig. 8. Response of vehicle states in accordance with measured vehicle speed-

TABLE V

INFINITE NoRm of Vehicle States REsponding to MeAsured VEHICLE SPEED

\begin{tabular}{|c|c|c|}
\hline Vehicle state & Type -1 controller & Type -2 controller \\
\hline Lateral offset & $3.35 e-02$ & $1.34 e-02$ \\
\hline Heading error & $2.19 e-03$ & $2.22 e-03$ \\
\hline Yaw rate & $1.12 e-02$ & $2.72 e-03$ \\
\hline Steering rate & $2.83 e-03$ & $2.38 e-03$ \\
\hline
\end{tabular}

parameters in the driver-automation shared driving system (11) are also challenges. Differing from the vehicle speed $v_{x}$, $K_{d 1}$ and $K_{d 2}$ are related to the driver behavior. In particular, $K_{d 1}$ represents the driver's proportional action on near angle and $K_{d 2}$ describes the driver's proportional action on far angle. They indicate the driver's contemporary behavior and anticipatory behavior, respectively. Generally, different drivers have differen parameters $K_{d 1}$ and $K_{d 2}$. In order to further demonstrate the superiority of type-2 fuzzy technology in dealing with high order uncertainties, an extensive simulation is tested, in which uncertain parameters $K_{d 1}$ and $K_{d 2}$ resulted from the diversity of drivers are considered.

As mentioned above, the vehicle longitudinal velocity $v_{x}$ and the weighting function $\mu\left(\theta_{d}(t)\right)$ in the driver-automation shared driving system (11) are time-varying. In addition, $K_{d 1}$ and $K_{d 2}$ are uncertain because of the driver diversity with the property of $K_{d i} \in\left[K_{\text {dimin }}, K_{\text {dimax }}\right], i=1,2$. There are five premise variables $v_{x}, \frac{1}{v_{x}}, \frac{K_{d 1}}{v_{x}}, \frac{K_{d 2}}{v_{x}}$ and $\mu\left(\theta_{d}(t)\right)$. Similar to the previous work, the variable $v$ is introduced to decouple variables $v_{x}, \frac{1}{v_{x}}, \frac{K_{d 1}}{v_{x}}$ and $\frac{K_{d 2}}{v_{x}}$. Then, the premise variables $\mu\left(\theta_{d}(t)\right), K_{d 1} v$ and $K_{d 2} v$ can be obtained. To reduce the number of premise variables ulteriorly, a new variable $K=K_{d 1} / K_{d 2}$ is introduced, which has the property of $K \in\left[K_{\min }, K_{\max }\right]$. It should be noted that there are $K>1$ and $K_{\min }=K_{d 1 \min } / K_{d 2 \max }, K_{\max }=K_{d 1 \max } / K_{d 2 \min }$. 
Consequently, only two premise variable $K v$ and $\mu\left(\theta_{d}(t)\right)$ are utilized. Then, the membership function could be defined as $f_{1}=K v$ and $f_{2}=\mu\left(\theta_{d}(t)\right)$. Following the same steps mentioned above, the extensive simulation results are depicted in Fig. 9 and Fig. 10.
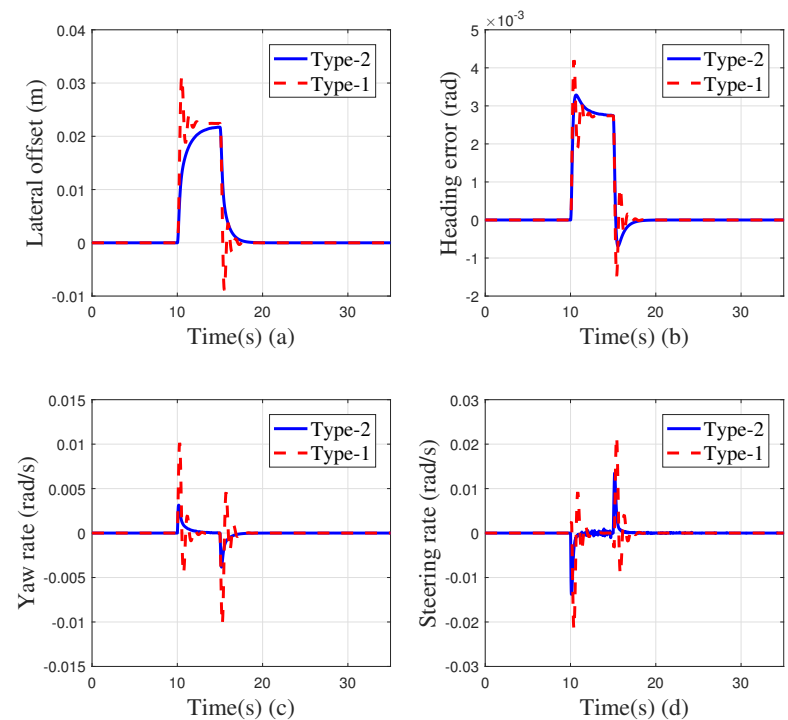

Fig. 9. Response of vehicle states in accordance with given road curvature-II.
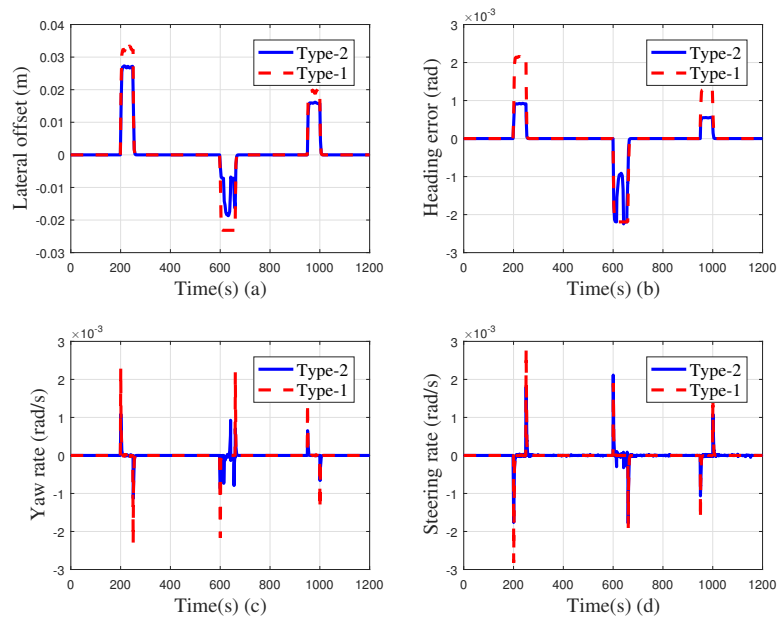

Fig. 10. Response of vehicle states in accordance with measured vehicle speed curvature-II.

Simulation results in Fig. 9 and Fig. 10 show that the type-2 fuzzy controller can promise smaller lateral offset and heading error, which means better lane keeping performance. Apart from it, as depicted in Fig. 9 (c) and Fig. 10 (c), the type-2 fuzzy controller produces lesser yaw rate compared to the type-1 fuzzy controller, indicating that it could promise better vehicle stability. Besides, a larger steering rate will influence human drivers when they put their hands on the steering wheel during the driving process. It can been seen from Fig. 9 (d) and Fig. 10 (d) that the type-2 fuzzy controller brings more modest effect on the human driver than the type1 fuzzy controller. In addition, results in Fig. 9 and Fig. 10 show that the type-2 fuzzy controller has better transient performance, as the type-2 fuzzy controller could converge to the steady-state quickly while the type-1 fuzzy controller has brief oscillation in the response process. It is because the $\mathcal{D}$-stability method is introduced in this work. As a result, it can be concluded that the type-2 fuzzy controller is superior to the type-1 fuzzy controller not only on the lane keeping performance and vehicle stability but also on the transient performance.

\section{Conclusions}

This paper investigates a driver-automation shared control system in the presence of imprecise vehicle speed. In fact, the vehicle longitudinal velocity is not only time-varying but also inaccurate. Meanwhile, the driver state $D S$ in the weighting parameter $\mu\left(\theta_{d}(t)\right)$ is not constant but time-variant. Both of them can cause uncertain membership function and if it is ignored when designing controller, the controller performance will be attenuated. In this case, the conventional type-1 T-S fuzzy technology is invalid to analyze the driver-automation shared control system in the presence of uncertain membership function. Therefore, a type-2 fuzzy technology is developed to address it, in which the upper and lower bounds of membership function are considered. Moreover, both stability and $\mathcal{H}_{\infty}$ performance are studied for the type-2 fuzzy driver-automation shared control system. The final simulation results also show advantages of the proposed type-2 fuzzy driver-automation shared controller, compared with the existing type-1 fuzzy controller. In the future research, more complex road condition will be considered to pursue better lane keeping performance. In addition, a practical experiment will be done to check and update the proposed algorithm.

\section{Declarations}

\section{Acknowledgements}

Not applicable.

\section{Author's contributions}

YL wtote the paper; QX reviesed the work; HG provided some ideas; HZ supervised the research.

\section{Author's information}

Hui Zhang is a professor with the department of Automotive Engineering, Beihang University, China.

\section{Funding}

Not applicable.

\section{Availability of data and materials}

Not applicable.

\section{Competing interests}

The authors declare no competing financial interests.

\section{Author Details}

Yue Liu, and Hui Zhang are with the School of Transportation Science and Engineering, Beihang University, Beijing 100091, China. e-mail: (sy1613207@buaa.edu.cn; Huizhang285@gmail.com) 
Qing $\mathrm{Xu}$ is with Department of automotive engineering, Tsinghua University, Beijing, China

Hong yan Guo is with Colleague of automotive engineering, Jilin University, Jilin, China

Received: XXXX Revised: XXXXX Accepted: XXXXX

\section{REFERENCES}

[1] J. C. F. D. Winter, R. Happee, M. H. Martens, and N. A. Stanton, "Effects of adaptive cruise control and highly automated driving on workload and situation awareness: A review of the empirical evidence," Transportation Research Part F Psychology \& Behaviour, vol. 27, pp. 196-217, 2014

[2] N. Merat, A. H. Jamson, F. C. Lai, and O. Carsten, "Highly automated driving, secondary task performance, and driver state." Human Factors, vol. 54, no. 5, pp. 762-771, 2012.

[3] J. Wu, S. Cheng, B. Liu, and C. Liu, "A human-machine-cooperativedriving controller based on AFS and DYC for vehicle dynamic stability," Energies, vol. 10, no. 11, p. 1737, 2017.

[4] Y. P. Kuo and T. S. Li, "GA-based fuzzy PI/PD controller for automotive active suspension system," IEEE Trans Industrial Electronic, vol. 46, no. 6, pp. 1051-1056, 1999.

[5] D. A. Abbink, T. Carlson, M. Mulder, J. C. de Winter, F. Aminravan, T. L. Gibo, and E. R. Boer, "A topology of shared control systems finding common ground in diversity," IEEE Transactions on HumanMachine Systems, vol. 48, no. 5, pp. 509-525, 2018.

[6] M. Johns, B. Mok, D. Sirkin, N. Gowda, C. Smith, W. Talamonti, and W. Ju, "Exploring shared control in automated driving," in $A C M / I E E E$ International Conference on Human-Robot Interaction, 2016, pp. 91-98.

[7] F. Flemisch, F. Nashashibi, N. Rauch, A. Schieben, S. Glaser, G. Temme, P. Resende, B. Vanholme, C. Loper, and G. Thomaidis, "Towards highly automated driving: Intermediate report on the HAVEit-Joint system," in Transport Research Arena, Brussels, 2010.

[8] M. R. Endsley and D. B. Kaber, "Level of automation effects on performance, situation awareness and workload in a dynamic control task," Ergonomics, vol. 42, no. 3, pp. 462-492, 1999.

[9] R. Li, S. Li, H. Gao, K. Li, B. Cheng, and D. Li, "Effects of human adaptation and trust on shared control for driver-automation cooperative driving," Tech. Rep. 2017-01-1987, 2017.

[10] L. Saleh, P. Chevrel, F. Claveau, J.-F. Lafay, and F. Mars, "Shared steering control between a driver and an automation: Stability in the presence of driver behavior uncertainty," IEEE Transactions on Intelligent Transportation Systems, vol. 14, no. 2, pp. 974-983, 2013.

[11] M. Huang, W. Gao, Y. Wang, and Z. Jiang, "Data-driven shared steering control of semi-autonomous vehicles," IEEE Transactions on HumanMachine Systems, vol. 49, no. 4, pp. 350-361, 2019.

[12] M. Li, H. Cao, G. Li, S. Zhao, X. Song, Y. Chen, and D. Cao, "A two-layer potential-field-driven model predictive shared control towards driver-automation cooperation," IEEE Transactions on Intelligent Transportation Systems, pp. 1-17, 2020.

[13] S. M. Erlien, S. Fujita, and J. C. Gerdes, "Shared steering control using safe envelopes for obstacle avoidance and vehicle stability," IEEE Trans. Intelligent Transportation Systems, vol. 17, no. 2, pp. 441-451, 2016.

[14] X. Ji, K. Yang, X. Na, C. Lv, and Y. Liu, "Shared steering torque control for lane change assistance: A stochastic game-theoretic approach," IEEE Transactions on Industrial Electronics, vol. 66, no. 4, pp. 3093-3105, 2019.

[15] R. Li, Y. Li, S. E. Li, C. Zhang, E. Burdet, and B. Cheng, "Indirect shared control for cooperative driving between driver and automation in steer-by-wire vehicles," IEEE Transactions on Intelligent Transportation Systems, pp. 1-11, 2020.

[16] Y. Lu, L. Bi, and H. Li, "Model predictive-based shared control for braincontrolled driving," IEEE Transactions on Intelligent Transportation Systems, vol. 21, no. 2, pp. 630-640, 2020.

[17] A. T. Nguyen, C. Sentouh, and J. C. Popieul, "Driver-automation cooperative approach for shared steering control under multiple system constraints: Design and experiments," IEEE Transactions on Industrial Electronics, vol. 64, no. 5, pp. 3819-3830, 2017.

[18] K. Tanaka and H. O. Wang, Fuzzy control systems design and analysis: a linear matrix inequality approach. John Wiley \& Sons, 2004.

[19] L. A. Zadeh, "The concept of a linguistic variable and its application to approximate reasoning - I," Information Sciences, vol. 8, no. 3, pp. 199-249, 1975.
[20] H. K. Lam and L. D. Seneviratne, "Stability analysis of interval type2 fuzzy-model-based control systems," IEEE Transactions on Systems Man \& Cybernetics Part B Cybernetics, vol. 38, no. 3, pp. 617-28, 2008.

[21] H. K. Lam, H. Li, C. Deters, E. L. Secco, H. A. Wurdemann, and K. Althoefer, "Control design for interval type-2 fuzzy systems under imperfect premise matching," IEEE Transactions on Industrial Electronics, vol. 61, no. 2, pp. 956-968, 2014.

[22] E. Kayacan, , and M. A. Khanesar, "Identification of nonlinear dynamic systems using type-2 fuzzy neural networks - A novel learning algorithm and a comparative study," IEEE Transactions on Industrial Electronics, vol. 62, no. 3, pp. 1716-1724, 2015

[23] D. Sun, Q. Liao, and H. Ren, "Type-2 fuzzy modeling and control for bilateral teleoperation system with dynamic uncertainties and timevarying delays," IEEE Transactions on Industrial Electronics, vol. 65, no. 1, pp. 447-459, 2018.

[24] N. N. Karnik, J. M. Mendel, and Q. Liang, "Type-2 fuzzy logic systems," IEEE Transactions on Fuzzy Systems, vol. 7, no. 6, pp. 643-658, 1999.

[25] O. Castillo and P. Melin, "A review on interval type-2 fuzzy logic applications in intelligent control," Information Sciences, vol. 279, pp. 615-631, 2014

[26] H. A. Hagras, "A hierarchical type-2 fuzzy logic control architecture for autonomous mobile robots," IEEE Transactions on Fuzzy Systems, vol. 12, no. 4, pp. 524-539, 2004.

[27] L. Zhang, H. K. Lam, Y. Sun, and H. Liang, "Fault detection for fuzzy semi-Markov jump systems based on interval type-2 fuzzy approach," IEEE Transactions on Fuzzy Systems, vol. 28, no. 10, pp. 2375-2388, 2020.

[28] T. Kumbasar and H. Hagras, "A self-tuning zslices-based general type2 fuzzy PI controller,' IEEE Transactions on Fuzzy Systems, vol. 23, no. 4, pp. 991-1013, 2015.

[29] P. K. Muhuri, Z. Ashraf, and Q. M. D. Lohani, "Multiobjective reliability redundancy allocation problem with interval type-2 fuzzy uncertainty," IEEE Transactions on Fuzzy Systems, vol. 26, no. 3, pp. 1339-1355, 2018.

[30] Y. Liu and H. Zhang, "Robust driver-automation shared control for a lane keeping system using interval type 2 fuzzy method," in 2019 IEEE 28th International Symposium on Industrial Electronics (ISIE). IEEE, 2019, pp. 1944-1949.

[31] R. Rajamani, Vehicle Dynamics and Control, 2006.

[32] J. Ackermann, J. Guldner, W. Sienel, R. Steinhauser, and V. I. Utkin, "Linear and nonlinear controller design for robust automatic steering," IEEE Transactions on Control Systems Technology, vol. 3, no. 1, pp. 132-143, March 1995.

[33] D. A. Abbink, M. Mulder, and E. R. Boer, "Haptic shared control: smoothly shifting control authority," Cognition, Technology \& Work, vol. 14, no. 1, pp. 19-28, 2012.

[34] H. Zhang, X. Zhang, and J. Wang, "Robust gain-scheduling energy-topeak control of vehicle lateral dynamics stabilisation," Vehicle System Dynamics, vol. 52, no. 3, pp. 309-340, 2014.

[35] J. H. Kim and B. P. Hong, " $\mathcal{H}_{\infty}$ state feedback control for generalized continuous/discrete time-delay system," Automatica, vol. 35, no. 8, pp. 1443-1451, 1999.

[36] N. M. Enache, M. Netto, S. Mammar, and B. Lusetti, "Driver steering assistance for lane departure avoidance," Control Engineering Practice, vol. 17, no. 6, pp. 642-651, 2009.

[37] M. Park, S. Lee, and W. Han, "Development of steering control system for autonomous vehicle using geometry-based path tracking algorithm," Eelctronics Telecommunications Research Inst, vol. 37, no. 3, pp. 617625, 2015.

[38] A. K. Madhusudhanan and X. Na, "Effect of a traffic speed based cruise control on an electric vehicle's performance and an energy consumption model of an electric vehicle," IEEE/CAA Journal of Automatica Sinica, vol. 7 , no. 2, pp. 386-394, 2020 


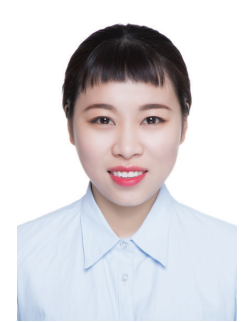

Yue Liu received the B.Sc. degree in automotive engineering from Henan University of Science and Technology, Luo Yang, China, in 2016. She is currently working toward the Ph.D. degree in vehicle dynamics at the School of Transportation Science and Engineering, Beihang University, Beijing, China.

Her research interests include vehicle dynamics and advanced control, automationdriver interaction, and multivehicle cooperative control problems.

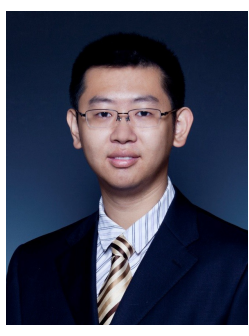

Qing Xu received his B.S. and M.S. degrees in automotive engineering from Beihang University, Beijing, China, in 2006 and 2008 respectively. During his Ph.D. research, he worked as a visiting scholar in department of mechanical science and engineering, UIUC, and got his Ph.D. degree in automotive engineering from Beihang University in 2014.

From 2014 to 2016, he had his postdoctoral research in Tsinghua University. And now he is working as an assistant research professor in department of automotive engineering, Tsinghua University. Qing Xu's main research interests include decision and control of intelligent vehicles.

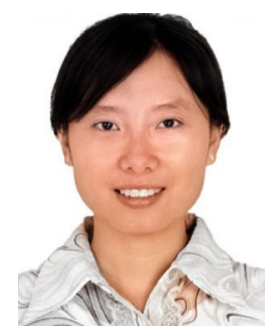

Hongyan Guo (M'17) received the PH.D. degree in control theory and control engineering from the Jilin University, Changchun, China, in 2010.

She joined the Jilin University, in 2011, where from 2014, she is an Associate Professor with the Department of Control Science and Engineering. In 2017, she is a Visiting Scholar with Cranfield University, Cranfield, U.K. Her current research interests include path tracking and stability control of autonomous vehicles and vehicle states estimation.

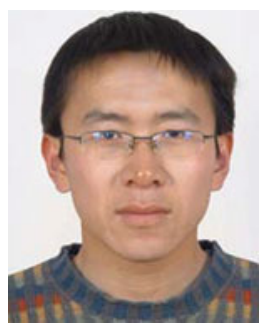

Hui Zhang (M'15-SM'17) received the B.Sc. degree in mechanical design manufacturing and automation from the Harbin Institute of Technology at Weihai,Weihai, China, in 2006, the M.Sc. degree in automotive engineering from Jilin University, Changchun, China, in 2008, and the Ph.D. degree in mechanical engineering from the University of Victoria, Victoria, BC, Canada, in 2012.

$\mathrm{He}$ is with the School of Transportation Science and Engineering, Beihang University, Beijing, China. His research interests include diesel engine aftertreatment systems, vehicle dynamics and control, mechatronics, robust control and filtering, networked control systems, and multi-agent systems. $\mathrm{He}$ is an author / co-author of more than 80 peer-reviewed papers on journals and conference proceedings. 